\title{
Levels and Values of Inflammatory Markers in Patients With Angina Pectoris
}

\author{
Hon-Kan YIP, ${ }^{1} \mathrm{MD}$, Chiung-Jen WU, ${ }^{1} \mathrm{MD}$, Chi-Ling HANG,${ }^{1} \mathrm{MD}$, \\ Hsueh-Wen CHANG,${ }^{2} \mathrm{PhD}$, Cheng-Hsu YANG, ${ }^{1} \mathrm{MD}$, Yuan-Kai HsIEH, ${ }^{1} \mathrm{MD}$, \\ Chih-Yuan FANG, ${ }^{1} \mathrm{MD}$, Morgan FU, ${ }^{1} \mathrm{MD}$, Kuo-Ho YeH, ${ }^{1} \mathrm{MD}$, \\ and Mien-Cheng CHEN, ${ }^{1} \mathrm{MD}$
}

\section{SUMMARY}

Inflammation plays an important pathogenic role in the initiation and progression of atherosclerotic plaque lesions. C-reactive protein (CRP), which directly participates in plaque inflammation, induces vascular cell adhesion molecule-1 (VCAM-1) expression in endothelial cells. However, the levels and values of high-sensitivity (hs)-CRP, white blood cell (WBC) count, and VCAM-1 in both stable and unstable angina pectoris (AP) have not been fully investigated. This study examines the levels and values of these inflammatory markers in patients with stable or unstable AP.

From March 2003 to December 2003, a prospective cohort study was conducted in 128 consecutive patients, including unstable AP patients (class I: $n=59$; combined class II and III: $n=16)$ and stable AP patients ( $n=53)$ undergoing elective coronary stenting. Blood samples for hs-CRP, WBC count, and VCAM-1 were obtained in the catheterization laboratory before coronary angiography. The circulating levels of hs-CRP and VCAM-1 were also evaluated in 40 healthy volunteers. The circulating levels of these three inflammatory markers were substantially higher in patients than in healthy volunteers (all $P$ values $<0.0001$ ). Additionally, circulating levels of hs-CRP and the WBC count were significantly higher in patients with unstable AP than in patients with stable AP (all $P$ value $<0.0001$ ). However, only those patients with class II and III unstable AP had significantly higher circulating levels of VCAM-1 than patients with stable AP $(P<$ 0.0001). On the other hand, the circulating levels of VCAM-1 did not differ between patients with class I unstable AP and patients with stable AP $(P=0.782)$. Multiple stepwise logistic regression analysis showed that only hs-CRP level was independently associated with unstable AP $(P=0.0002)$. In conclusion, circulating levels of hs-CRP, WBC count, and VCAM-1 were significantly increased in patients with AP. The circulating level of hs-CRP was strongly associated with the clinical setting of unstable AP. (Int Heart J 2005; 46: 571-581)

Key words: Unstable angina, Obstructive coronary artery disease, Inflammatory markers

From the ${ }^{1}$ Division of Cardiology, Chang Gung Memorial Hospital, Kaohsiung, ${ }^{2}$ Department of Biological Sciences, National Sun Yat-Sen University, Kaohsiung, Taiwan, R.O.C.

Address for correspondence: Mien-Cheng Chen, MD, Division of Cardiology, Department of Internal Medicine, Chang Gung Memorial Hospital, Kaohsiung, 123 Ta Pei Road, Niao Sung Hsiang, Kaohsiung Hsien, 83301, Taiwan, R.O.C.

Received for publication January 5, 2005.

Revised and accepted February 18, 2005. 
Atherosclerosis is a dynamic and progressive disease arising from the combination of endothelial dysfunction and inflammation. ${ }^{1-3)}$ Inflammation where leukocytes adhere to endothelial cells, followed by an interaction between these cells mediated by inflammatory mediators and inflammatory cytokines, has been shown to play a crucial role in all stages of atherosclerosis from initiation, through to progression, and finally acute coronary syndromes. ${ }^{1,4-6)} \mathrm{C}$-reactive protein (CRP), having recently emerged as an important inflammatory mediator, could directly participate in the pathogenesis of atherosclerosis by activating endothelial cells and promoting the inflammatory component of atherosclerosis. ${ }^{7,8)}$ Additionally, CRP has been demonstrated to provoke expression of adhesion molecules and chemokines in human endothelial cells. ${ }^{9)}$ Furthermore, the interaction of these expressed adhesion molecules and their ligands has been shown to mainly govern leukocyte and endothelial cell adhesion during the inflammatory process. ${ }^{7,8)}$

Some recent studies have demonstrated that higher levels of serum CRP are related to increased risk of coronary events in patients with stable ${ }^{10)}$ and unstable angina, ${ }^{10,11)}$ and are related to untoward short-term and long-term outcomes in patients with non-ST segment elevated ${ }^{1)}$ and ST-segment elevated ${ }^{13)}$ acute coronary syndromes. However, the levels and values of inflammatory markers in patients with AP remain less clear.

Taken together, we hypothesized that the circulating levels of three inflammatory markers: white blood cell (WBC) count, high-sensitivity (hs)-CRP, and vascular cell adhesion molecule-1 (VCAM-1) are significantly higher in AP patients and remarkably higher in unstable AP than stable AP patients and normal subjects.

\section{METHODS}

Patient population and inclusion criteria: This study included consecutive patients admitted for unstable or stable angina to our hospital between March 2003 and December 2003. For the purpose of this study, the serum levels of hs-CRP, WBC count, and VCAM-1 of all patients who underwent coronary stenting were prospectively measured. Blood samples were drawn after vascular puncture before coronary angiography was performed in the cardiac catheterization room and in our outpatient department on day 21 after the procedure. To avoid other variables that could influence the serum levels of hs-CRP, the WBC count, and VCAM-1, we excluded patients with a history of recent surgery or trauma within the preceding 2 months, renal insufficiency (creatinine $>1.5 \mathrm{mg} / \mathrm{dL}$ ), malignancy or liver cirrhosis, febrile disorders, acute or chronic inflammatory disease at study entry, history of recent infection, as well as those with acute myocardial infarc- 
tion (AMI) onset of $<3$ months. The obstruction had to be a new lesion for which the patient had not undergone prior coronary angioplasty. Patients were excluded if they were receiving adjunctive tirofiban, clopidogrel, or statin therapy preceding blood sampling because the potential impact of these drugs on hs-CRP and VCAM-1 could not be determined. Patients were also excluded if fever (body temperature $>37.5^{\circ} \mathrm{C}$ ) was observed before cardiac catheterization. Over a period of 10 months, we prospectively recruited 128 consecutive patients (53 patients with stable AP and 75 patients with unstable AP) who underwent stenting on a single nonocclusive coronary stenosis. In addition, 40 age- and gender-matched nonhospitalized healthy volunteers were studied as a control group. Blood samples were obtained once in healthy volunteers at the out-patient department. Informed consent was obtained from all study subjects. The study protocol was approved by the Institutional Review Committee on Human Research at our institution.

Procedure and protocol: A transradial artery approach using a 6-F arterial sheath and a 6-F Kimny Miniradi (Boston Scientific, Scimed, Maple Grove, MN) was routinely utilized for both coronary angiographic study and percutaneous coronary intervention (PCI) in this study, except where Allen's test was positive.

Clopidogrel (300 mg loading dose after stenting followed by $75 \mathrm{mg} /$ day) was administered for at least four weeks to our patients following stenting and aspirin (100 mg orally once daily) was administered to each patient indefinitely. Laboratory investigations: WBC cells were counted and biochemical measurements were made using standard laboratory methods. The hs-CRP was measured by immunonephelometry using the $\mathrm{BN}^{\mathrm{TM}}$ system (Dade Behring Inc. Newark, DE, USA). The lower detection limit of this test is $<0.15 \mathrm{mg} / \mathrm{L}$ and a serum hsCRP level of $1.2 \mathrm{mg} / \mathrm{L}$ was taken to be the upper high normal limit in our hospital laboratory. We assessed the intraindividual variability of serum hs-CRP levels in study patients and healthy subjects. The mean intra-assay coefficients of variance were $3.21 \%$ and $2.96 \%$, respectively.

The concentration of VCAM-1 was measured using a standard enzymelinked immunosorbent assay (ELISA) and a commercially available kit (Diaclone; Besancon, France). The ELISA method is described in detail in our recent report. ${ }^{14)}$ The assay was sufficiently sensitive to detect less than $0.6 \mathrm{ng} / \mathrm{mL}$ of VCAM-1, according to the manufacturer of the assay kits. The intraindividual variability of VCAM-1 levels was assessed in study patients and healthy subjects. In our laboratory, the mean intra-assay coefficients of variation were $<9 \%$.

Definitions and data collection: Multivessel disease was defined as stenoses of $>$ $50 \%$ in $\geq 2$ major epicardial coronary arteries. A combined 30-day major cardiac event was defined as recurrent ischemia, new onset of AMI, repeated target vessel revascularization, or 30-day death. Unstable AP was defined (according to 
Braunwald's classification $)^{15)}$ as the presence of one or more of the following three historical features: 1) crescendo angina superimposed on a preexisting pattern or relatively stable, exertion-related AP; 2) AP of new onset, which was brought on by minimal exertion; or 3) AP at rest as well as with minimal exertion. The severity of unstable AP was also categorized into three classifications: ${ }^{15)} 1$ ) class I: new onset, severe, or accelerated angina (patients with angina of less than 2 month's duration, no rest pain in the last two months); 2) class II: angina occurring at rest and > 48 hours; 3) class III: angina occurring at rest and within 48 hours.

Detailed in-hospital data including age, sex, coronary risk factors, medical history, characteristics of chest pain, ECG findings, body temperature on admission, creatinine level, WBC count, cardiac isoenzyme and troponin levels before cardiac catheterization, angiographic findings, and number of diseased vessels were obtained. These data were collected prospectively and entered into a computerized database.

Statistical analysis: Categorical variables were compared using the chi-square test or Fischer's exact test. Univariate analyses were performed using Student's $t$ test. Continuous variables were compared using the Wilcoxon rank-sum test. Continuous variables among the three groups were compared using one-way ANOVA for parametric data and the Kruskal-Wallis test with the Bonferroni correction for nonparametric data. Statistical analysis was performed using SAS statistical software for Windows version 8.2 (SAS Institute, Cary, NC). A probability value $<0.05$ was considered statistically significant.

\section{ReSults}

Baseline characteristics of study patients and healthy control subjects (Table I): Table I summarizes the baseline characteristics of the stable angina patients, unstable angina patients, and normal control subjects. The three groups did not differ significantly in age, gender, body mass index, or creatinine levels. There were no significant differences in hypertension, diabetes mellitus, current smoking, hypercholesterolemia, previous MI, previous stroke, or multivessel coronary artery disease between patients with stable AP and patients with unstable AP.

Comparison of circulating levels of inflammatory markers between unstable angina patients, stable angina patients, and normal control subjects (Tables II and III): Table II displays the circulating levels of hs-CRP, WBC counts, and VCAM-1 in the stable AP and unstable AP patients, and normal control subjects. The circulating level of hs-CRP did not differ between the stable angina patients and normal control subjects $(P>0.5)$. However, the circulating level of hs-CRP was significantly higher in patients with unstable AP than in either stable AP patients or nor- 
Table I. Baseline Characteristics of Study Patients and Normal Control Subjects

\begin{tabular}{lcccc}
\hline \multicolumn{1}{c}{ Variables } & $\begin{array}{c}\text { Stable angina } \\
(n=53)\end{array}$ & $\begin{array}{c}\text { Unstable angina } \\
(n=75)\end{array}$ & $\begin{array}{c}\text { Normal control } \\
(n=40)\end{array}$ & $P$ value \\
\hline Age (years) (mean $\pm \mathrm{SD})$ & $59.9 \pm 11.0$ & $62.2 \pm 10.4$ & $59.8 \pm 8.6$ & 0.283 \\
Male gender & $81.1 \%(43)$ & $77.3 \%(58)$ & $60.0 \%(24)$ & 0.051 \\
Hypertension & $62.3 \%(33)$ & $57.3 \%(43)$ & - & 0.576 \\
Diabetes mellitus & $34.0 \%(18)$ & $25.3 \%(19)$ & - & 0.289 \\
Current smoker & $35.9 \%(19)$ & $46.7 \%(35)$ & - & 0.222 \\
Hypercholesterolemia & $37.7 \%(20)$ & $48.0 \%(36)$ & - & 0.249 \\
Previous myocardial infarction & $18.9 \%(10)$ & $24.0 \%(18)$ & - & 0.489 \\
Previous stroke & $5.7 \%(3)$ & $2.7 \%(2)$ & - & 0.648 \\
Body mass index $\left(\mathrm{kg} / \mathrm{m}^{2}\right)$ & $27.4 \pm 5.5$ & $25.7 \pm 3.3$ & $26.8 \pm 4.6$ & 0.471 \\
Creatinine (mg/dL) & $1.08 \pm 0.25$ & $1.05 \pm 0.25$ & $1.07 \pm 0.31$ & 0.862 \\
Multivessel disease & $73.6 \%(39)$ & $61.3 \%(46)$ & - & 0.148 \\
\hline
\end{tabular}

Values are given as mean value \pm SD or \% (no. of patients), unless otherwise indicated.

Table II. Comparison of Circulating Levels of Inflammatory Markers Between Study Patients and Normal Control Subjects

\begin{tabular}{lcccc}
\hline \multicolumn{1}{c}{ Variable } & $\begin{array}{c}\text { Stable angina } \\
(n=53)\end{array}$ & $\begin{array}{c}\text { Unstable angina } \\
(n=75)\end{array}$ & $\begin{array}{c}\text { Normal control } \\
(n=40)\end{array}$ & $P$ value \\
& $1.48 \pm 1.26^{*}$ & $3.40 \pm 2.93 \dagger$ & $1.08 \pm 0.74^{*}$ & $<0.0001$ \\
Hs-CRP $(\mathrm{mg} / \mathrm{L})$ & $6.63 \pm 1.57 \ddagger$ & $7.07 \pm 2.02 \ddagger$ & $5.11 \pm 0.88 \S$ & $<0.0001$ \\
WBC count $\left(\times 10^{3} / \mathrm{mL}\right)$ & $737.6 \pm 359.4 \llbracket$ & $771.1 \pm 342.7 \Phi$ & $533.0 \pm 68.4 \|$ & $<0.0001$ \\
VCAM-1 $(\mathrm{ng} / \mathrm{mL})$ & & &
\end{tabular}

Values are given as mean value $\pm \mathrm{SD}$.

Hs-CRP = high-sensitivity C-reactive protein; VCAM-1 = vascular cell adhesion molecule-

$1 ; \mathrm{WBC}=$ white blood cell

Symbols $(*, \dagger, \ddagger, \S$, II, and $\|)$ indicate significance (at level 0.05 ) by Wilcoxon rank-sum test with Bonferroni correction.

$\dagger$ versus $*, P<0.0001$.

† versus $\S, P<0.0001$.

II versus $\|, P<0.0001$.

mal control subjects. Both the stable and unstable AP patients had significantly higher circulating levels of WBC and VCAM-1 than normal control subjects. Nevertheless, there was no difference in the circulating levels of either of these two inflammatory markers between the stable and unstable AP patients.

To test whether the baseline characteristics and the three inflammatory markers differed between patients with class II and III unstable AP and patients with both class I unstable AP and stable AP, we further analyzed these three groups of patients (Table III). The baseline characteristics, angiographic findings, and WBC counts did not significantly differ among these patients. The circulating level of hs-CRP did not differ between patients with class I AP and patients 
Table III. Comparison of Baseline Characteristics and Laboratory Findings Among Patients With Stable Angina, Class I Unstable Angina, and Combined Class II and III Unstable Angina

\begin{tabular}{lcccc}
\hline \multicolumn{1}{c}{ Variable } & $\begin{array}{c}\text { Unstable angina } \\
\text { (Class I)* }(n=59)\end{array}$ & $\begin{array}{c}\text { Unstable angina } \\
\text { Class II and III)* } \\
(n=16)\end{array}$ & $\begin{array}{c}\text { Stable angina } \\
(n=53)\end{array}$ & $P$ value \\
\hline Age (years) (mean $\pm \mathrm{SD})$ & $61.6 \pm 11.3$ & $64.6 \pm 6.3$ & $59.9 \pm 11.07$ & 0.308 \\
Male gender & $72.9 \%(43)$ & $93.8 \%(15)$ & $81.8 \%(43)$ & 0.168 \\
Hypertension & $57.6 \%(34)$ & $56.3 \%(9)$ & $62.3 \%(33)$ & 0.851 \\
Diabetes mellitus & $25.4 \%(15)$ & $25.0 \%(4)$ & $34.0 \%(18)$ & 0.569 \\
Current smoker & $35.9 \%(19)$ & $50.9 \%(30)$ & $31.3 \%(5)$ & 0.176 \\
Hypercholesterolemia & $49.2 \%(29)$ & $43.8 \%(7)$ & $37.7 \%(20)$ & 0.477 \\
Previous myocardial infarction & $22.0 \%(13)$ & $31.3 \%(5)$ & $18.9 \%(10)$ & 0.831 \\
Previous stroke & $3.4 \%(2)$ & $0 \%(0)$ & $5.7 \%(3)$ & 0.576 \\
Body mass index $\left(\mathrm{kg} / \mathrm{m}^{2}\right)$ & $25.8 \pm 3.3$ & $25.5 \pm 3.6$ & $27.4 \pm 5.5$ & 0.269 \\
Creatinine $(\mathrm{mg} / \mathrm{dL})$ & $1.04 \pm 0.24$ & $1.09 \pm 0.27$ & $1.08 \pm 0.25$ & 0.810 \\
Multivessel disease & $61.0 \%(36)$ & $62.5 \%(6)$ & $73.6 \%(39)$ & 0.350 \\
WBC count $\left(\times 10^{3} / \mathrm{mL}^{2}\right)$ & $7.11 \pm 2.17$ & $6.94 \pm 1.38$ & $6.63 \pm 1.57$ & 0.554 \\
VCAM-1 $(\mathrm{ng} / \mathrm{mL})$ & $702.1 \pm 321.5 \dagger$ & $1025.4 \pm 303.4 \ddagger$ & $737.8 \pm 359.4 \dagger$ & 0.0002 \\
Hs-CRP $(\mathrm{mg} / \mathrm{L})$ & $3.23 \pm 2.61 \S$ & $4.01 \pm 3.95 \S$ & $1.48 \pm 1.26 \Phi$ & 0.0003 \\
Combined 30-day MACE* & $0 \%(0)$ & $0 \%(0)$ & $0 \%(0)$ & 1.000 \\
\hline
\end{tabular}

Values are given as mean value $\pm \mathrm{SD}$ or $\%$ (no. of patients). Hs-CRP $=$ high-sensitivity C-reactive protein; MACE $=$ major adverse cardiac events; VCAM = vascular cell adhesion molecule; $\mathrm{WBC}=$ white blood cells. * Definition of unstable angina is described in text. Symbols $(\dagger, \ddagger, \S$, and II) indicate significance (at level 0.05 ) by Wilcoxon rank-sum test with Bonferroni correction.

$\ddagger$ versus $\dagger, P=0.0002$. § versus $\mathbb{I}, P=0.0003$.

with class II and III AP. Moreover, the circulating level of VCAM-1 did not significantly differ between patients with class I AP and patients with stable AP. Nevertheless, the circulating level of VCAM-1 was substantially higher in patients with class II and III unstable AP than in both class I AP and stable AP patients. Furthermore, the circulating levels of these three inflammatory markers on day 21 following PCI were repeatedly measured in the study patients, with the results showing that the circulating levels of VCAM-1 (756.8 \pm 334.4 versus $741.5 \pm 365.4$ versus $714.3 \pm 322.1, P=0.712)$, hs-CRP $(2.29 \pm 2.55$ versus 1.95 \pm 1.74 versus $1.57 \pm 1.79, P=0.231)$, and $\mathrm{WBC}(6.8 \pm 1.4$ versus $6.7 \pm 2.0$ versus $6.3 \pm 1.4, P=0.248$ ) did differ among these three groups of patients. Moreover, no major adverse cardiac event was observed at 30-day clinical follow-up.

Multiple stepwise logistic regression analysis of the baseline and clinical variables at enrolment (Table III) demonstrated that only hs-CRP was significantly independently associated with unstable angina (odds ratio $=1.56,95 \%$ confidence interval: $1.23-2.0, P=0.0002$ ). 
Table IV. Comparison of Circulating Levels of Inflammatory Markers Between Multivessel and Single Vessel Disease

\begin{tabular}{lccc}
\hline \multicolumn{1}{c}{ Variable } & $\begin{array}{c}\text { Multivessel disease } \\
(\mathrm{n}=85)\end{array}$ & $\begin{array}{c}\text { Single vessel disease } \\
(\mathrm{n}=43)\end{array}$ & $P$ value \\
& $2.50 \pm 2.60$ & $2.82 \pm 2.48$ & 0.313 \\
Hs-CRP $(\mathrm{mg} / \mathrm{L})$ & $7.00 \pm 0.97$ & $6.67 \pm 1.61$ & 0.408 \\
WBC count $\left(\times 10^{3} / \mathrm{mL}\right)$ & $767.2 \pm 319.6$ & $752.2 \pm 364.3$ & 0.208 \\
VCAM-1 & & \\
\hline Values are given as mean value \pm SD. & & \\
Hs-CRP = high-sensitivity C-reactive protein; VCAM-1 = vascular cell adhesion \\
molecule-1; WBC = white blood cell.
\end{tabular}

Comparison of circulating levels of inflammatory markers between study patients and normal control subjects (Table IV): Table IV lists the circulating levels of inflammatory markers in patients with multivessel disease and in patients with single vessel disease. There were no significant differences in hs-CRP, WBC, or VCAM-1 between patients with multivessel disease and patients with single vessel disease.

\section{DiscuSSION}

This study, in which the circulating levels of inflammatory markers were examined in patients with AP undergoing elective PCI, produced three major findings. First, circulating levels of hs-CRP and WBC were distinctively higher in patients with unstable AP than in patients with stable AP. Second, enhanced expression of circulating VCAM-1 mainly occurred in the early stage of unstable AP. This finding, therefore, suggests that markedly increased circulating levels of VCAM-1 only appear following an acute inflammatory stimulus. Third, circulating levels of hs-CRP were strongly correlated to unstable AP.

Circulating levels and impact of hs-CRP on coronary artery atherosclerotic disease in stable and unstable angina: Previous studies have demonstrated that higher levels of serum CRP correlate with increased risk of coronary events in patients with stable and unstable angina. ${ }^{10,11)}$ Our recent study demonstrated that circulating levels of hs-CRP were significantly higher in patients with an onset of AMI < $6 \mathrm{~h}$ than in healthy subjects and patients with stable AP undergoing elective PCI. ${ }^{13)}$ This study demonstrated that patients with unstable AP had significantly increased circulating levels of hs-CRP compared to those patients with stable AP. Furthermore, the circulating level of hs-CRP was independently related with the clinical setting of unstable AP. The findings are consistent with previous studies $^{10,11)}$ and our recent ${ }^{13)}$ study. The findings further strengthen the suggestion 
that hs-CRP may not only mirror an inflammatory stimulus, but also directly promote atherosclerotic propagation and destabilization of plaque. ${ }^{13)}$

Recent studies have demonstrated that CRP is related to adverse outcomes in patients with non-ST-segment elevation acute coronary syndromes. ${ }^{12,16)}$ Even after early aggressive coronary intervention, the serum level of CRP remains a strong independent predictor of short ${ }^{17)}$ and long-term mortality in these patients. ${ }^{12)}$ Systemic inflammation and widespread inflammatory processes throughout the coronary vessel, as reflected by elevated levels of CRP, have been recognized as contributing to untoward short- and long-term outcomes. ${ }^{16,18)}$ The presence of multiple complex coronary plaques ${ }^{18)}$ and multivessel disease are angiographic hallmarks of diffuse coronary disease. A striking association between multivessel disease and poor clinical outcomes has been established. ${ }^{12,19-22)}$ Surprisingly, this investigation failed to demonstrate a link between circulating levels of hs-CRP and multivessel disease. Interestingly, two previous studies ${ }^{23,24)}$ have also demonstrated that CRP is not strongly and independently associated with prevalent atherosclerosis. Therefore, our results are comparable with previous studies. $^{23,24)}$

From the findings we postulate that assessing the impact of the changes and the raised circulating levels of hs-CRP on coronary plaque instability in patients with acute coronary syndrome may provide better correlation rather than focusing on the relationship between levels of hs-CRP and multivessel disease. This suggestion is upheld by recent studies ${ }^{25,26)}$ which have demonstrated that the rise in hs-CRP is associated with a thinning fibrous cap and the presence of ruptured plaque, resulting in AMI and death from acute coronary syndromes. In aggregate, our laboratory and angiographic findings, intravascular ultrasound observations, ${ }^{26)}$ and findings of immunohistochemical staining in other research ${ }^{25)}$ may raise suspicions that rupture of an atherosclerotic plaque could be, at least in part due to the sharply increasing plasma level of hs-CRP. This mechanistic hypothesis of plaque rupture resembles the mechanistic basis of ovulation following a luteinizing hormone surge in the female. Further research is therefore encouraged to ascertain whether serial measurement of changes in hs-CRP in patients with coronary artery disease could yield more valuable clinical information for future strategic management of these patients in this setting.

In the present study, the rapid decline in the circulating level of hs-CRP (on day 21) after stenting in patients with unstable AP could be due to two reasons. The first is that CRP is an acute-phase reactant. Therefore, by its natural course, it will decline after an acute inflammatory stimulation. The second would be due to successful stenting to the plaque lesion which was the origin of the inflammatory site. 
Association between increased circulating level of WBC and unstable angina: Increased circulating levels of WBC, an index of inflammation, have been shown to be related to increased adverse clinical outcomes in patients with acute coronary syndromes. ${ }^{27)}$ Other studies ${ }^{28,29)}$ have further demonstrated that an elevated WBC count on admission is associated with reduced epicardial flow and myocardial reperfusion, thromboresistance, postprocedural thrombus burden, large infarct size, and a higher incidence of reinfarction after PCI for AMI. These data imply that patients with a higher baseline WBC count have a heightened inflammatory process. ${ }^{28,29)}$ The present study showed that WBC count was noticeably higher in unstable AP than in stable AP patients. These findings are in agreement with previous studies, ${ }^{27-29)}$ and therefore, further reinforce the recommendation from previous investigators ${ }^{28)}$ that WBC count is a new and more straightforward tool for risk stratification for AMI and unstable AP.

Link between increased circulating VCAM-1 and unstable angina: CRP has been demonstrated to increase the expression of VCAM-1 and intercellular adhesion molecule-1 in both human umbilical vein and coronary artery endothelial cells. $^{9,30)}$ The increased expression of both adhesion molecules promotes the adhesion of monocytes which then migrate into the subendothelial space and augment vascular smooth muscle cell proliferation. ${ }^{8)}$ These studies suggest that CRP may directly promote the inflammatory component of atherosclerosis. ${ }^{8,9,30)}$ Therefore, it is no surprise that VCAM-1 and other adhesion molecules are considered as important biomarkers of inflammation. ${ }^{8)}$ In the present study, the circulating levels of VCAM-1 did not vary in patients with unstable versus stable AP. However, patients with Class II and III unstable AP had substantially higher circulating levels of VCAM-1 than patients with either Class I unstable or stable AP. Furthermore, circulating levels of hs-CRP did not differ between patients with Class II and III and patients with Class I unstable angina. Several reasons could explain this discrepancy. First, we suggest that enhanced expression of VCAM-1 could only be augmented in the early stage of acute inflammatory stimulus by a sharply increasing hs-CRP. Second, we suggest that this finding may reflect different inherent pathways between VCAM-1 and hs-CRP for the response of an acute inflammatory stimulus. This hypothesis is based on differences in cellular sources of these inflammatory biomarkers. It is well recognized that CRP, an acute inflammatory reactant, is primarily synthesized and secreted rapidly in the liver after an acute inflammatory stimulus. On the other hand, VCAM-1 is mainly derived from vascular endothelial cells as a result of a stimulation of elevated CRP levels, or other factors which could cause endothelial cell dysfunction such as ischemia. Third, after an obstructive ischemia was resolved by coronary stenting, the endothelial cell function would be quickly improved. This could in turn, 
lead to reduce the stimulation of endothelial cells to inappropriately secrete the VCAM-1 and other cytokines.

Study limitations: First, a six-month angiographic follow-up was not completely assessed in the present study. Therefore, this investigation did not address how circulating levels of hs-CRP and VCAM-1 impact late restenosis. Second, the inferences made concerning the severity of coronary atherosclerosis in this study are based on the angiographic findings of single versus multivessel disease, but not on intravascular ultrasound studies for the number of plaque lesions or the complexity of the stenosis. The sensitivity of intravascular ultrasound may reinforce the claim of an association between the severity of coronary disease and circulating levels of hs-CRP and VCAM-1. Hence, the sensitivity herein of the diagnosis of severity of coronary disease may not be optimal.

In conclusion, circulating levels of hs-CRP and WBC were significantly higher in patients with unstable AP than in patients with stable AP and normal control subjects. Circulating levels of hs-CRP were independently associated with unstable AP. Enhanced expression of circulating VCAM-1 mainly occurred in an early stage of AP. Lack of an association between increased circulating levels of inflammatory markers and multivessel disease was found in unstable and stable angina. The results of this investigation suggest that increased circulating levels of hs-CRP and VCAM-1 appear more sensitive at determining the stage of the inflammatory process than to predicting the severity of coronary artery disease.

\section{ACKNOWLEDGMENTS}

The authors wish to thank Ms. Hsium-Chin Tsai, Fang-Yu Dai, and Chia-Jung Lin for their technical assistance.

\section{REFERENCES}

1. Ross R. Atherosclerosis-an inflammatory disease. N Engl J Med 1999; 340: 115-26. (Review)

2. Libby P, Ridker PM, Maseri A. Inflammation and atherosclerosis. Circulation 2002; 105: 1135-43. (Review)

3. Libby P. Inflammation in atherosclerosis. Nature 2002; 420: 868-74. (Review)

4. Ross R. The pathogenesis of atherosclerosis: a prospective for the 1990s. Nature 1993; 362: 802-9. (Review)

5. Hansson GK. Immune and inflammatory mechanisms in the development of atherosclerosis. Br Heart J 1993; 69: S38-41. (Review)

6. van der Wall AC, Becker AE, van der Loos CM, Das PK. Site of intimal rupture or erosion of thrombosed coronary atherosclerotic plaques is characterized by an inflammatory process irrespective of the dominant plaque morphology. Circulation 1994; 89: 36-44.

7. Verma S, Buchanan MR, Anderson TJ. Endothelial function testing as a biomarker of vascular disease. Circulation 2003; 108: 2054-9. (Review)

8. Szmitko PE, Wang CH, Weisel RD, de Almeida JR, Anderson TJ, Verma S. New markers of inflammation and endothelial cell activation: Part I. Circulation 2003; 108: 1917-23. (Review) 
9. Pasceri V, Willerson JT, Yeh ET. Direct proinflammatory effect of C-reactive protein on human endothelial cells. Circulation 2000; 102; 2165-8.

10. Haverkate F, Thompson SG, Pyke SD, Gallimore JR, Pepys MB. Production of C-reactive protein and risk of coronary events in stable and unstable angina. European Concerted Action on Thrombosis and Disabilities Angina Pectoris Study Group. Lancet 1997; 349: 462-6.

11. Liuzzo G, Biascucci LM, Gallimore JR, et al. The prognostic value of C-reactive protein and serum amyloid a protein in severe unstable angina. N Eng J Med 1994; 331: 417-24.

12. Mueller C, Buettner HJ, Hodgson JM, et al. Inflammation and long-term mortality after non-ST elevation acute coronary syndrome treated with a very early invasive strategy in 1042 consecutive patients. Circulation 2002; 105: 1412-5.

13. Yip HK, Wu CJ, Chang HW, et al. Levels and values of serum high-sensitivity C-reactive protein within six hours after the onset of an acute myocardial infarction Chest 2004; 126: 1414-22.

14. Chen MC, Chang HW, Juang SS, et al. Percutaneous transluminal mitral valvuloplasty reduces circulating vascular cell adhesion molecule-1 in rheumatic mitral stenosis. Chest 2004; 125: 1213-7.

15. Braunwald E. Unstable angina. A classification. Circulation 1989; 80: 410-4.

16. Lindahl B, Toss H, Siegbahn A, Venge P, Wallentin L. Markers of myocardial damage and inflammation in relation to long-term mortality in unstable coronary artery disease. FRISC Study Group. Fragmin during Instability in Coronary Artery Disease. N Engl J Med 2000; 343: 1139-47.

17. Yip HK, Wu CJ, Chang HW, et al. Prognostic value of circulating levels of endothelin-1 in patients after acute myocardial infarction undergoing primary coronary angioplasty. Chest 2005; 127: 1491-7.

18. Goldstein JA, Demetriou D, Grines CL, Pica M, Shoukfeh M, O'Neill WW. Multiple complex coronary plaques in patients with acute myocardial infarction. N Engl J Med 2000; 343: 915-22.

19. Hannan EL, Racz MJ, Arani DJ, Ryan TJ, Walford G, McCallister BD. Short- and long-term mortality for patients undergoing primary angioplasty for acute myocardial infarction. J Am Coll Cardiol 2000; 36: 1194201.

20. Yip HK, Wu CJ, Yang CH, et al. Delayed post-myocardial infarction invasive measures, helpful or harmful? A subgroup analysis. Chest 2004; 126: 38-46.

21. Kennedy J W, Kaiser GC, Fisher LD, et al. Clinical and angiographic predictors of operative mortality from the collaborative study in coronary artery surgery (CASS). Circulation 1981; 63: 793-802.

22. Yip HK, Wu CJ, Chang HW, et al. Comparison of impact of primary percutaneous transluminal coronary angioplasty and primary stenting on short-term mortality in patients with cardiogenic shock and evaluation of prognostic determinants. Am J Cardiol 2001; 87: 1184-8.

23. Redberg RF, Rifai N, Gee L, Rider PM. Lack of association of C-reactive protein and coronary calcium by electron beam computed tomography in postmenopausal women: implications for coronary artery disease screening. J Am Coll Cardiol 2000; 36: 39-43.

24. Folsom AR, Pankow JS, Tracy RP, et al. Association of C-reactive protein with markers of prevalent atherosclerotic disease. Am J Cardiol 2001; 88: 112-7.

25. Burke AP, Tracy RP, Kologie F, et al. Elevated C-reactive protein values and atherosclerosis in sudden coronary death: association with different pathologies. Circulation 2002; 105: 2019-23.

26. Sano T, Tanaka A, Namba M, et al. C-reactive protein and lesion morphology in patients with acute myocardial infarction. Circulation 2003; 108: 282-5.

27. Cannon CP, McCabe CH, Wilcox RG, Bentley JH, Braunwald E. Association of white blood cell count with increased mortality in acute myocardial infarction and unstable angina pectoris. OPUS-TIMI 16 Investigators. Am J Cardiol 2001; 87: 636-9.

28. Barron HV, Cannon CP, Murphy SA, Braunwald E, Gibson CM. Association between white blood cell count, epicardial blood flow, myocardial perfusion, and clinical outcomes in the setting of acute myocardial infarction: a thrombolysis in myocardial infarction 10 substudy. Circulation 2000; 102: 2329-34.

29. Pellizzon GG, Dixon SR, Stone GW, et al. Relation of admission white blood cell count to long-term outcomes after primary coronary angioplasty for acute myocardial infarction (The Stent PAMI Trial). Am J Cardiol 2003; 91: 729-31.

30. Verma S, Li SH, Badiwala MV, et al. Endothelial antagonism and interleukin-6 inhibition attenuate the proatherogenic effects of C-reactive protein. Circulation 2002; 105: 1890-6. 